\title{
Successful outcomes of a new combined solution of hyaluronic acid, chondroitin sulfate and poloxamer 407 for submucosal injection: animal survival study
}

\section{(ㄷ)(1) $(-)$}

\author{
Authors \\ Institutions \\ 1 Endoscopy Unit, Gastroenterology Department, \\ ICMDiM, IDIBAPS, CIBEREHD, Hospital Clínic, Universitat \\ de Barcelona, Barcelona, Spain \\ 2 Pathology Department, CDB, Hospital Clínic, IDIBAPS, \\ Universitat de Barcelona, Banc de Tumors Biobanc \\ Clinic-IDIBAPS, Barcelona, Spain \\ 3 Anesthesiology Department, Hospital Clínic, Universitat \\ de Barcelona, Barcelona, Spain
}

Henry Córdova ${ }^{1}$, Miriam Cuatrecasas², Ana García-Rodríguez ${ }^{1}$, Andrea Montenegro¹, Jordana Melo', Cristina Rodríguez-de Miguel ${ }^{1}$, Graciela Martínez-Pallí ${ }^{3}$, Rodrigo Garcés-Durán ${ }^{1}$, Josep Llach ${ }^{1}$, Gloria Fernández-Esparrach ${ }^{1}$

submitted 27.9.2028

accepted after revision: 7.1 .2019

\author{
Bibliography \\ DOI https://doi.org/10.1055/a-0869-7757 | \\ Endoscopy International Open 2019; 07: E576-E582 \\ (c) Georg Thieme Verlag KG Stuttgart · New York \\ ISSN 2364-3722
}

Corresponding author

G. Fernández-Esparrach, Villarroel 170, 08036 Barcelona, Spain

Fax: +34932279387

mgfernan@clinic.ub.es
ABSTRACT

Background and study aims Endoscopic resection requires use of submucosal injection. This study was conducted to assess efficacy and impact on early healing of hyaluronic acid combined with chondroitin sulfate and poloxamer 407 (Ziverel) when used as a solution for submucosal injection.

Materials and methods Prospective and comparative study of gastric endoscopic mucosal resection (EMR) with three groups of two Yorkshire pigs. Six submucosal cushions were created in each animal by injecting $2 \mathrm{~mL}$ of Ziverel (Group 1) or succinylated gelatin (SG) (Group 2), enabling 12 EMR in each group. Submucosal cushions were created with Ziverel in Group 3, without resection. Electrosurgery unit settings were the same in all cases. EMR defects and injection sites were marked with clips. The animals were sacrificed 7 days later. EMR specimen size and duration of procedure were recorded. EMR specimens and EMR scars and injection sites were evaluated by a blinded pathologist.

Results We successfully performed 24 EMR (15 en-bloc and 9 piecemeal, without differences between groups 1 and 2). Mean EMR specimen dimensions were significantly larger in Group 1 (median $19 \mathrm{~mm}$, range $6-40$ vs $16.6 \mathrm{~mm}$, range $5-25 ; P=0.019$ ), without changing the electrocautery unit settings. Blinded histopathologist assessment of EMR specimens showed less fibrosis in the submucosa and a trend to fewer cautery artifacts with Ziverel and did not identify any significant differences in early healing of resection sites.

Conclusion The combination of Ziverel enables EMR and does not negatively affect early healing.

\section{Introduction}

Endoscopic mucosal resection (EMR) and endoscopic submucosal dissection (ESD) are widely used in the West as therapeutic methods for resection of non-polypoid lesions of the gastrointestinal tract. The most effective and simplest way to prevent adverse events during EMR and ESD is to create a sufficiently thick submucosal layer by endoscopic injection of fluid into the submucosa [1]. Sodium hyaluronic acid is commonly used in Japan to carry out ESD whereas in Western countries different solutions are available [2,3].

In a recent comparative study of the submucosal injection fluids currently available in the West, a volume expander (6\% hydroxyethyl starch) and Eleview (a mixture of medium chain triglycerides, poloxamer 188, polyoxyl-15-hydroxystearate, sodium chloride and methylene blue) were the best-performing 
solutions for ESD in a porcine model [4]. Moreover, an ideal solution should be inexpensive and without potential damage of the resected specimen or early healing impairment.

Recently, we have reported that a new treatment for gastroesophageal reflux that combines hyaluronic acid, chondroitin sulfate and poloxamer 407 (Ziverel) could be used as a solution for submucosal injection because it creates a long-lasting cushion, does not induce acute tissue damage and it is not expensive [5].

The objective of this study was to assess the efficacy and impact on early healing of Ziverel as submucosal injection solution for EMR compared with succinylated gelatin (SG), which is the standard solution used in our Unit.

\section{Materials and methods}

This prospective and comparative study was conducted with six Yorkshire pigs allocated into three groups: EMR with Ziverel (Norgine, UK) (Group 1, $n=2$ ), EMR with SG (Gelafundin, Braun Medical, Spain) (Group 2, $n=2$ ) and injection of Ziverel without resection (Group 3, $n=2$ ). Gastroscopy was conducted with the animals under general anesthesia and all the procedures were performed by a single endoscopist who was not blinded to which solution was injected.

Six submucosal cushions were created in each animal by injecting $2 \mathrm{~mL}$ of solution with a 23-gauge catheter injection needle (Interject, Boston Scientific Corp., Marlborough, Massachusetts, United States). Ziverel was diluted with saline solution at a concentration of $50 \%$ to ease the injection [4]. In Groups 1 and 2, a total of 24 EMR were performed (12 in each group) using a 25-mm polypectomy snare (SD-210U-25, Olympus Medical Systems Corp., Tokyo, Japan). Each EMR was the largest possible safe en-bloc or piecemeal resection. An ERBE electrosurgery unit was used (VIO 300 D, ERBE Co, Tübingen Germany) with the same settings (ENDOCUT Q mode, effect 3, duration 1, interval 6) in all cases. A 2-mL injection volume was chosen because preliminary experimentation showed that a larger volume resulted in a bleb that did not fit into the snare. At the end of the procedure, EMR defects and injection sites were marked with clips ( $\triangleright$ Fig. 1 ) and retrieved EMR specimens were pinned to a cork board ( $>$ Fig. 2 ).

EMR specimen size was measured (maximal length $\times$ maximal width) and EMR duration was measured by time and number of pulses of Endocut needed to completion of snare resection. Note was made of systematic adverse effects, bleeding or perforation.

Animals were observed daily for 6 days and were sacrificed on day 7 . The stomachs were retrieved and opened to find the remaining clips, and abdominal cavities were inspected. Stomachs and EMR specimens were fixed with formalin. Selected sections from each area marked with clips or mucosal lesion were cut and processed to obtain formalin-fixed paraffin-embedded tissue blocks. From each paraffin block, 2- $\mu$ m-thick histological sections were stained with hematoxylin and eosin (H\&E) and examined with a conventional Olympus BX41 microscope (Olympus, Tokyo, Japan) by a pathologist blinded to the solution used. Pathological features such as reepithelization was scored as $0=$ absence and $1=$ presence, while fibrosis and

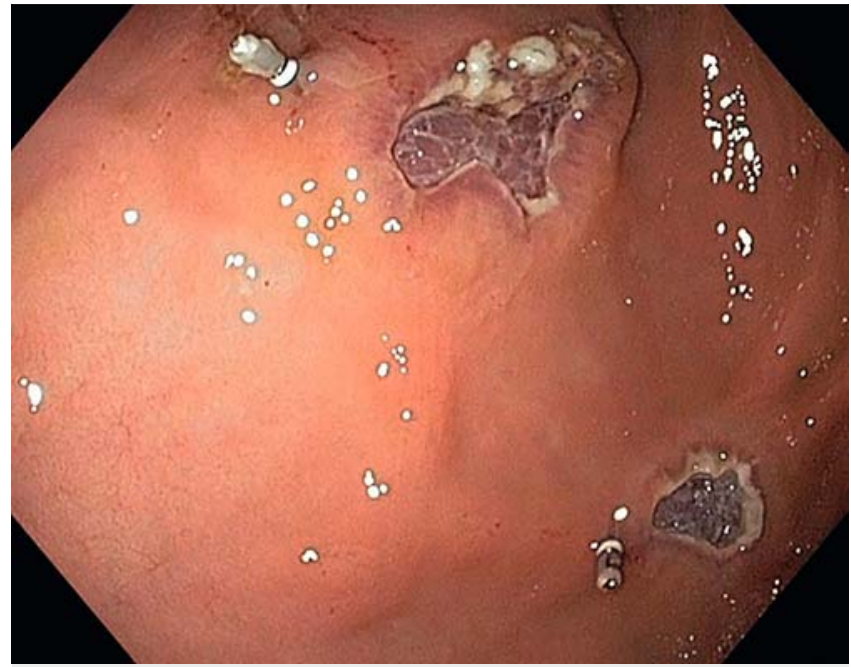

- Fig. 1 EMR defects and injection sites marked with clips.

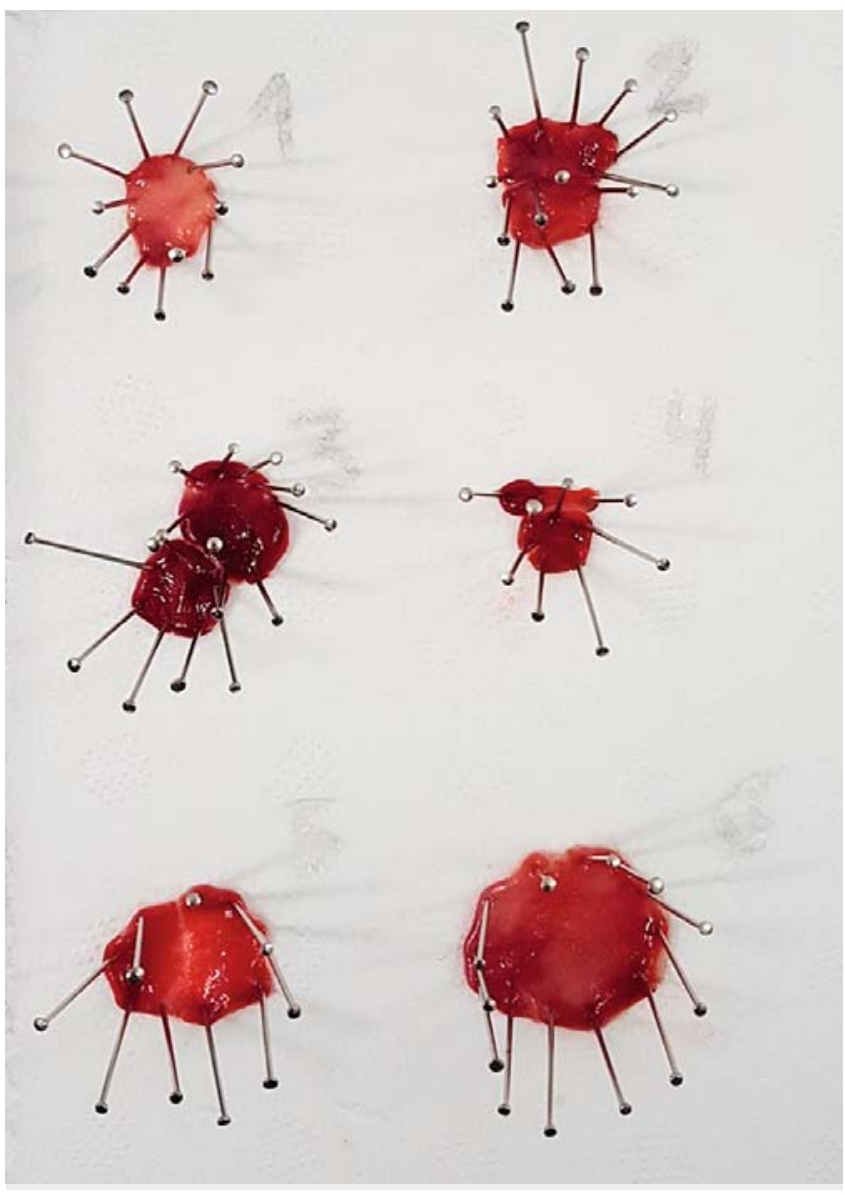

- Fig. 2 Retrieved EMR specimens pinned to cork.

acute and chronic inflammation were semi-quantitatively scored as 0 = absence; 1 mild; 2 intermediate; 3 high. Thickness of the submucosa in the resected specimen and the granulation tissue were measured in $\mathrm{mm}$.

The study was approved by the Ethics Animal Committee of Barcelona University. 
- Table 1 Technical and macroscopic characteristics of EMR.

\begin{tabular}{|c|c|c|c|c|c|c|}
\hline & \multicolumn{2}{|l|}{$\begin{array}{l}\text { Group } 1 \\
\text { (Ziverel }^{R} \text { ) }\end{array}$} & \multicolumn{2}{|l|}{$\begin{array}{l}\text { Group } 2 \\
\text { (SG) }\end{array}$} & \multicolumn{2}{|l|}{$P$ value } \\
\hline & $\begin{array}{l}\text { All EMR } \\
N=12\end{array}$ & $\begin{array}{l}\text { En-bloc } \\
\mathrm{N}=7\end{array}$ & $\begin{array}{l}\text { All EMR } \\
N=12\end{array}$ & $\begin{array}{l}\text { En-bloc } \\
\mathrm{N}=8\end{array}$ & All & En-bloc \\
\hline Maximal length (mm) & $19(6-40)$ & $19(17-40)$ & $16.5(5-25)$ & $16.5(5-22)$ & 0.019 & 0.035 \\
\hline Maximal width (mm) & $16(5-32)$ & $15(13-32)$ & $12.5(3-19)$ & $10(3-19)$ & 0.060 & 0.041 \\
\hline Time (minutes) & $10.6(1.5-19.7)$ & $8.5(1.5-19.7)$ & $10.2(3.4-19.7)$ & $9.2(3.4-19.7)$ & 0.95 & 0.972 \\
\hline Endocut pulses & $9.5(3-15)$ & $7(3-15)$ & $8.5(4-18)$ & $7.5(4-18)$ & 0.8 & 0.994 \\
\hline
\end{tabular}

\section{Statistical analysis}

Quantitative variables are expressed as median plus range. Statistical comparisons between groups 1 and 2 were made by using the Mann-Whitney non-parametric test. A $P$ value $<0.05$ was considered significant. Calculations were performed with SPSS software (SPSS 21.0 for Windows, SPSS, Inc., Chicago, Illinois, United States).

\section{Results}

We successfully performed 24 EMRs ( 15 en-bloc and 9 piecemeal). The median EMR specimen maximal length was significantly larger in Group 1 than in Group $2(19 \mathrm{~mm}$, range $6-40$ vs $16.5 \mathrm{~mm}$, range $5-25 ; P=0.019)$. Using the same electrocautery unit settings, mean time upon completion of all EMR was not different between groups ( $\triangleright$ Table 1 ). No perforation or intraprocedural hemorrhage was observed. Animals recovered well and gained weight (median $29.4 \mathrm{~kg}$, range $27.9-$ 20.6 vs $32 \mathrm{~kg}$, range $29.9-33.9$ ).

Microscopic examination of the EMR specimens showed a thicker submucosa with edema and a trend to fewer cauteryrelated artifacts in Group 1 ( $\vee$ Table 2, $>$ Fig. 3 ). The muscularis propria was not visible in any specimen.

We could identify the $24 \mathrm{EMR}$ scars in the gastrectomy specimens at the time of necropsy. Histological assessment of the scars did not show any significant differences between Group 1 and 2, except for more fibrotic changes in the latter ( $\triangleright$ Table 3 , - Fig. 4). With regards to the stomachs in Group 3, only mild fibrosis and chronic inflammation were identified. These findings are commonly described in pigs' normal gastric mucosa $(\triangleright$ Table 4).

\section{Discussion}

In this study, we compared a new treatment for gastroesophageal reflux that combines hyaluronic acid, chondroitin sulfate, and poloxamer 407 with a common solution used for submucosal injection and evaluated their impact on the outcomes of gastric EMR and early healing in pigs. Use of the new substance was associated with larger and deeper EMR specimens and few- er cautery artifacts, and did not negatively affect early healing or cause any long-term injury in the normal gastric wall.

SG was used as a control injection substance because it is used for performing EMR in our unit due to its durability, wide availability in our country and low price. Moreover, SG provides EMR specimens with larger surface area than with normal saline solution [3]. Compared with SG, Ziverel provided a better circumscribed elevation that facilitated tissue capture with the snare and a larger specimen.

The capability of Ziverel to separate the mucosal layer from the muscularis propria by increasing the thickness of the submucosa due to edema was already demonstrated in the pilot study performed by our group [5]. Poloxamer 407, which is one of the compounds of Ziverel, is a reverse phase polymer that becomes gel at body temperature, breaking and replacing the connective tissue. Having a thick submucosa layer included in the resected specimen is of paramount importance because it increases the chance of performing a curative resection with free margins, even in the case of deep infiltration of the superficial submucosa.

It is of crucial importance to achieve en bloc resection of colorectal laterally spreading tumors or early gastrointestinal neoplasia, because en bloc resection is associated with a low local recurrence rate $[6,7]$. In the last few years, endoscopic submucosal dissection (ESD) has emerged as an alternative to EMR when lesions are big and it is not possible to perform en bloc resections. However, piecemeal EMR is still the standard of care in several countries, but even in that case, it is preferable to obtain large specimens to decrease the number of pieces [8]. Although with Ziverel the rate of en bloc EMR was not different from SG, the size of the resected specimens was larger.

Damage to the resected specimen, either by piecemeal resection or by cauterization artifacts, can limit precise histological diagnosis of a targeted lesion, and hyaluronic acid has shown to be the least harmful [9]. Furthermore, damage to the muscle layer may result in delayed bleeding or perforation. The addition of poloxamer to hyaluronic acid used in the current study did not damage the mucosa of the EMR specimen, confirming its safety, but even more important was the fact that cautery damage of the borders was less evident than with SG.

The impact of potential solutions for submucosal injection on resected ulcer healing has been evaluated in several studies 


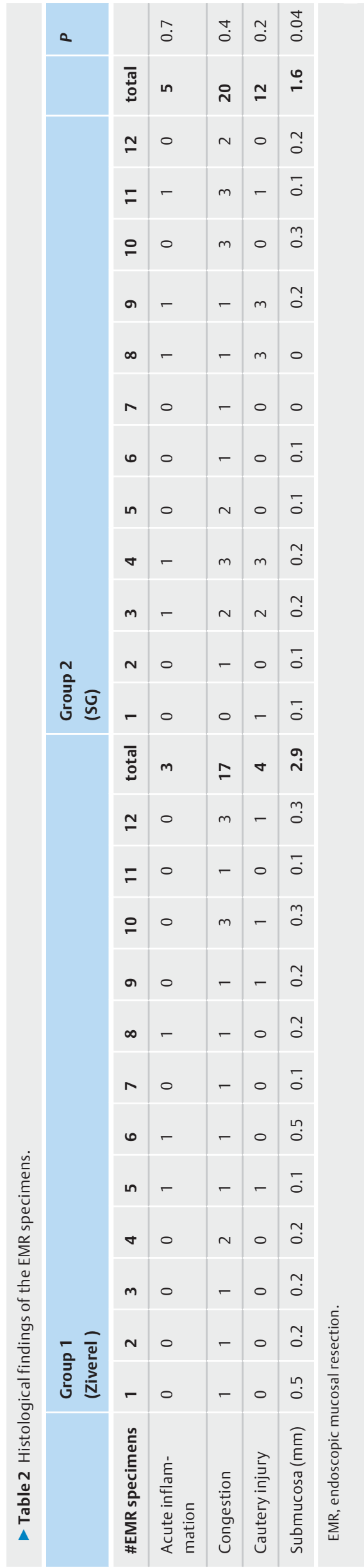

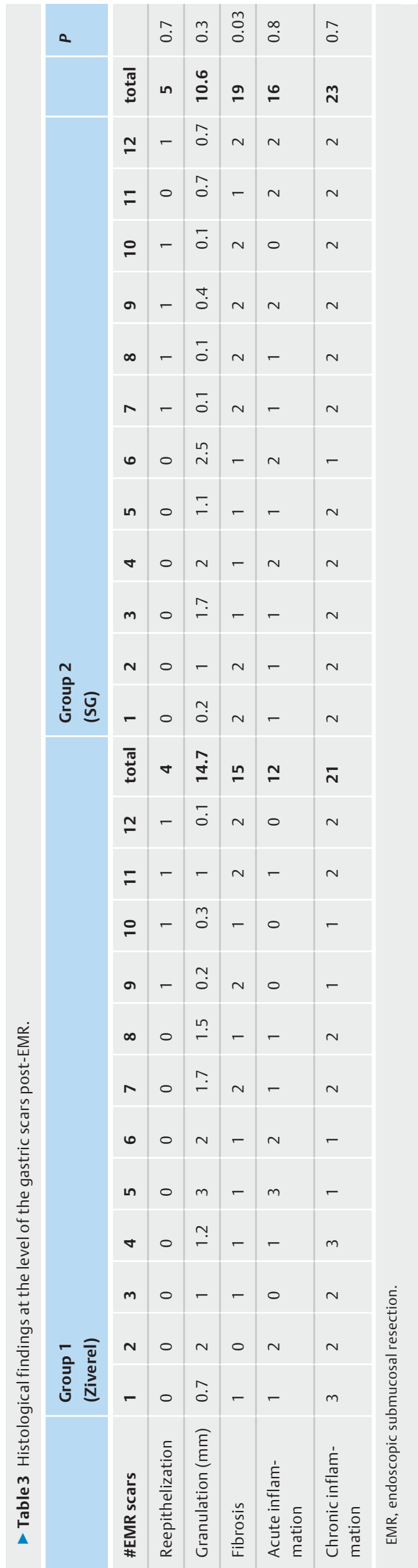



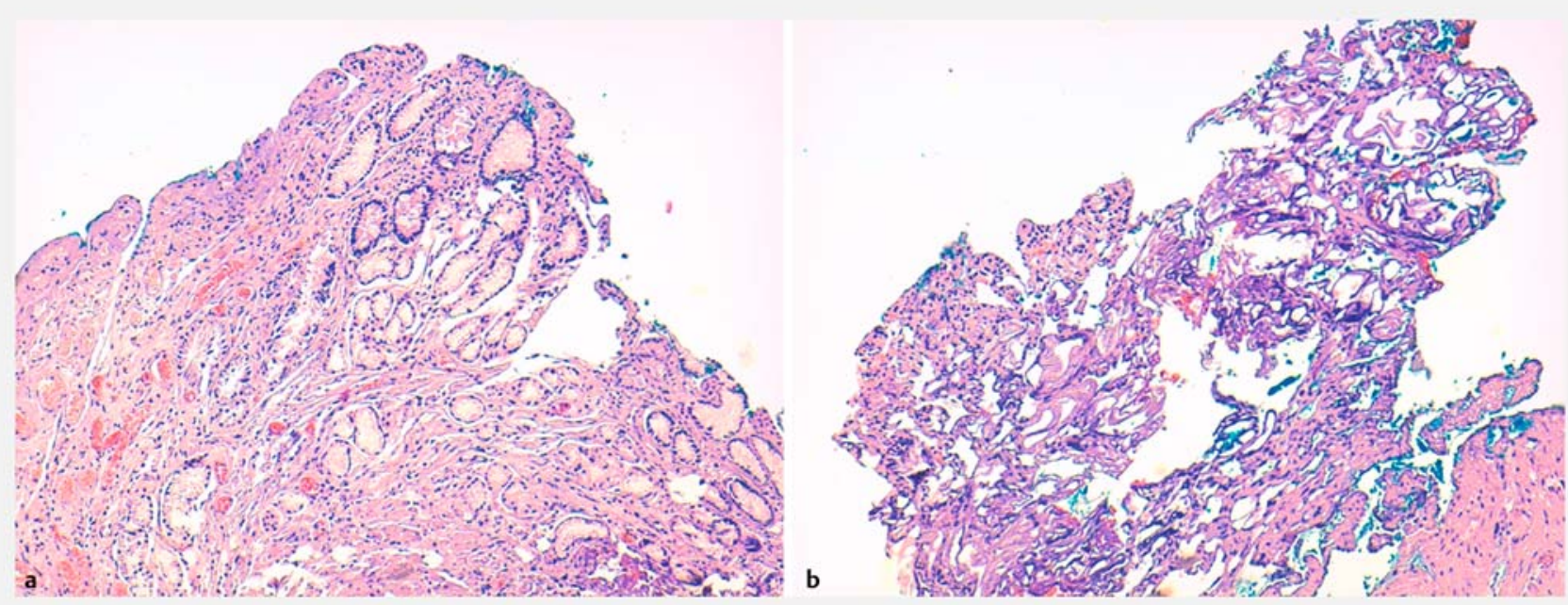

- Fig. 3 a Microscopic examination of the EMR specimens showing less mucosal cautery-related artifacts in the Ziverel group b than with SG, where the epithelial glands are not visible and architecture is lost. The muscularis propria is not visible in any specimen.
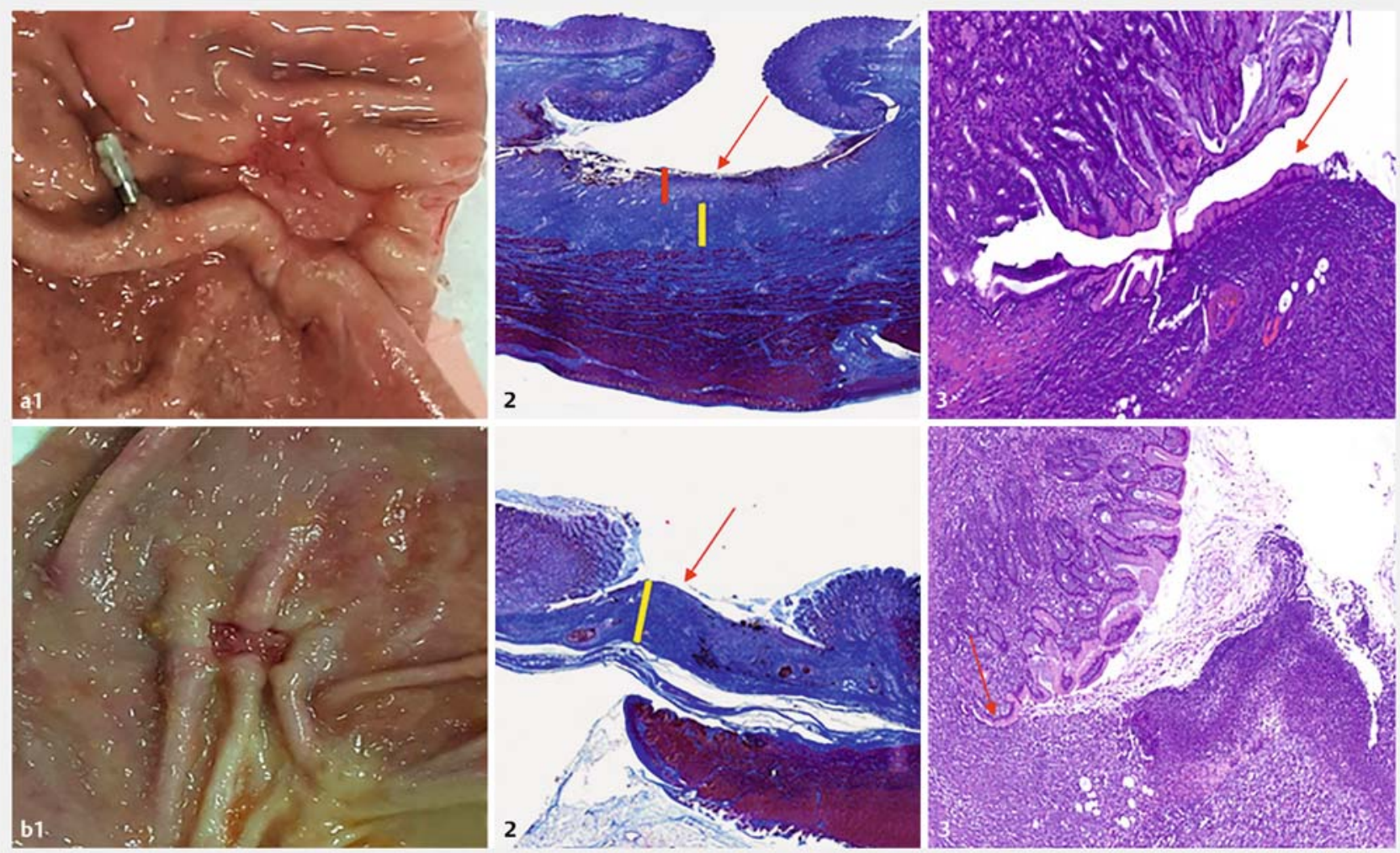

- Fig. 4 a (1) Gross appearance of the scars with larger mucosectomy area with Ziverel b than SG. a (2) Microscopic examination of the scars red arrows- shows residual superficial edema (red line) and less submucosal fibrosis (yellow line) with Ziverel b compared with SG. In this latter, there is no residual edema and thicker and more fibrotic changes in the submucosal scar. 3) Reepithelization is seen in both groups (red arrows), being more evident in a Ziverel group.

and glycerol has shown more pronounced histological signs of early healing [10]. In our study, Ziverel showed an early healing that was no different from SG, except for less fibrosis that could be explained by changes in permeability of injured mucosa
[11]. Based on these properties, we hypothesize that Ziverel could also facilitate the healing process of the scar left after EMR or ESD and expect an early proliferation of collagen and elastic fibers as described with other solutions [10,12]. 
- Table4 Histological findings in the stomachs injected with Ziverel without resection.

Group 3

(Ziverel ${ }^{\mathrm{R}}$ without resection)

\begin{tabular}{|c|c|c|c|c|c|c|c|c|c|c|c|c|c|}
\hline \# Injections & 1 & 2 & 3 & 4 & 5 & 6 & 7 & 8 & 9 & 10 & 11 & 12 & total \\
\hline Reepithelization & 0 & 0 & 0 & 0 & 0 & 0 & 0 & 0 & 0 & 0 & 0 & 0 & 0 \\
\hline Granulation (mm) & 0 & 0 & 0 & 0 & 0 & 0 & 0 & 0 & 0 & 0 & 0 & 0 & 0 \\
\hline Fibrosis & 1 & 1 & 1 & 1 & 1 & 1 & 1 & 0 & 0 & 0 & 1 & 2 & 10 \\
\hline Acute inflammation & 0 & 0 & 0 & 0 & 0 & 0 & 0 & 0 & 0 & 0 & 0 & 1 & 1 \\
\hline Chronic inflammation & 0 & 1 & 1 & 1 & 1 & 2 & 2 & 1 & 2 & 1 & 3 & 3 & 18 \\
\hline
\end{tabular}

The use of solutions with high osmolality, such as hypertonic saline solution or $20 \%$ to $50 \%$ dextrose water, induces acute mucosal damage consisting of erosion and congestion of capillary blood vessels that is persistent a week after injection, in the form of submucosal fibrosis [13]. Ziverel is also a hyperosmotic solution with around 2,000 mOsm/L but contrarily, was not associated with any significant abnormality in the gastric wall 1 week after the injection.

Another interesting result is that the new solution did not affect the speed of EMR. Using the same settings of the electrosurgical unit as with SG, there were no differences regarding in time needed to complete EMR. These results are consistent with the initial experience with poloxamer [14].

Among available submucosal injection solutions in clinical practice, so far the most suitable for producing a long-lasting cushion is high-molecular-weight hyaluronic solution [15]. However, the crucial disadvantage of hyaluronic acid in current formulations is the high cost, which limits its use in many countries. Therefore, the combination of hyaluronic acid with Glyceol has been proposed as an acceptable low-cost alternative [15]. Interestingly, the cost of Ziverel is significantly lower than hyaluronic acid alone ( 0.10 and 11.84 USD per $\mathrm{mL}$, respectively) and very similar to Gelafundin ( $0.08 \mathrm{USD}$ per $\mathrm{mL}$ ). Therefore, its use could decrease the final cost of the procedure, making its use widely available.

This study has the strength that the pathologist was blind to the solution used. The endoscopist knew which solution was tested, as noticeable differences in viscosity of the two solutions during the injection and the consistency of the blebs made endoscopist blinding impractical.

With regard to limitations, we only injected $2 \mathrm{~mL}$ of solution, which is far from the usual amount needed for a large EMR. We chose this amount because the consistency of the bleb made it impossible to catch a larger bleb with a $25-\mathrm{mm}$ snare.

\section{Conclusion}

In conclusion, the current study demonstrates that the combination of hyaluronic acid, chondroitin sulfate, and poloxamer 407 enables EMR and does not negatively affect early healing.

\section{Acknowledgement}

This work was supported by CERCA Programme/Generalitat de Catalunya.

\section{Competing interests}

Dr. Fernández-Esparrach has received fees for organizing courses for Norgine Iberia and Olympus Spain in the last 2 years and has been a consultant for a trial design for CDx Diagnostics

\section{References}

[1] Hwang JH, Konda V, Abu Dayyeh BK et al. Endoscopic mucosal resection. Gastrointest Endosc 2015; 82: 215-226

[2] Ferreira A, Moleiro J, Torres J et al. Solutions for submucosal injection in endoscopic resection: a systematic review and meta-analysis. Endosc Int Open 2015; 04: E1 - E16

[3] Moss A, Bourke M], Kwan V et al. Succinylated gelatin substantially increases en bloc resection size in colonic EMR: a randomized, blinded trial in a porcine model. Gastrointest Endosc 2010; 71: 589-595

[4] Mehta N, Strong AT, Franco M et al. Optimal injection solution for endoscopic submucosal dissection: A randomized controlled trial of Western solutions in a porcine model. Dig Endosc 2018; 30: 347- 353

[5] Fernández-Esparrach G, Cuatrecasas M, Rodríguez de Miguel C et al. Efficacy and safety of a combination of hyaluronic acid, chondroitin sulfate, and poloxamer 407 as a submucosal injection solution for endoscopic resection: pilot study on a swine model. Endosc Int Open 2017; 5: E450-E454

[6] Oka S, Tanaka S, Saito Y et al. Local recurrence after endoscopic resection for large colorectal neoplasia: a multicenter prospective study in Japan. Am J Gastroenterol 2015; 110: 697 - 707

[7] Belderbos T, Leenders M, Moons L et al. Local recurrence after endoscopic mucosal resection of nonpedunculated colorectal lesions: systematic review and meta-analysis. Endoscopy 2014; 46: 388-402

[8] Ferlitsch M, Moss A, Hassan C et al. Colorectal polypectomy and endoscopic mucosal resection (EMR): European Society of Gastrointestinal Endoscopy (ESGE) Clinical Guideline. Endoscopy 2017; 49: $270-297$

[9] Yamamoto H, Kawata $H$, Sunada K et al. Successful en-bloc resection of large superficial tumors in the stomach and colon using sodium hyaluronate and small-caliber-tip transparent hood. Endoscopy 2003; 35: $690-694$ 
[10] Bureš ], Kopáčová M, Květina J et al. Different solutions used for submucosal injection influenced early healing of gastric endoscopic mucosal resection in a preclinical study in experimental pigs. Surg Endosc Other Interv Tech 2009; 23: 2094-2101

[11] Di Simone M, Baldi F, Vasina $V$ et al. Barrier effect of Esoxx\&amp;reg; on esophageal mucosal damage: experimental study on ex-vivo swine model. Clin Exp Gastroenterol 2012: 103

[12] Uraoka T, Ochiai Y, Fujimoto A et al. A novel fully synthetic and selfassembled peptide solution for endoscopic submucosal dissectioninduced ulcer in the stomach. Gastrointest Endosc 2016; 83: 12591264
[13] Fujishiro M, Yahagi N, Kashimura K et al. Tissue damage of different submucosal injection solutions for EMR. Gastrointest Endosc 2005; 62: $933-942$

[14] Fernández-Esparrach G, Shaikh SN, Cohen A. Efficacy of a reversephase polymer as a submucosal injection solution for EMR: a comparative study (with video). Gastrointest Endosc 2009; 69: 1135 1139

[15] Fujishiro M, Yahagi N, Kashimura K et al. Comparison of various submucosal injection solutions for maintaining mucosal elevation during endoscopic mucosal resection. Endoscopy 2004; 36: 579-583 